\title{
A European questionnaire survey on epilepsy monitoring units' current practice for postoperative psychogenic nonepileptic seizures' detection
}

Sofia Markoula ${ }^{\mathrm{a}, 1}$, Andreas Liampas ${ }^{\mathrm{a}, 1}$, Guido Rubboli ${ }^{\mathrm{b}}$, John Duncan ${ }^{\mathrm{c}}$, Demetrios N Velis ${ }^{d}$, Andreas Schulze-Bonhage ${ }^{\mathrm{e}}$, Alla Guekht ${ }^{\mathrm{f}}$, Karen Bartholomeyczik ${ }^{\mathrm{e}}$, Jane de Tisi ${ }^{\mathrm{c}}$, Adrien Gras ${ }^{g}$, Morten Ingvar Lossius ${ }^{h}$, Flavio Villani', Staack Anke Maren', Annette Hospes $^{d}$, Johannes C. Baaijen ${ }^{d}$, Elisabeth C.W. van Straaten ${ }^{d}$, Hanneke E. Ronner ${ }^{d}$, Sara

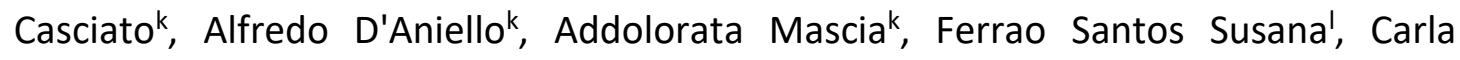
Bentes $^{m}$, Ángel Aledo-Serrano ${ }^{n}$, Antonio Gil-Nagel ${ }^{n}$, Petia Dimova ${ }^{\circ}$, Hrvoje Hećimović p, Çiğdem Özkaraq ${ }^{q}$ Kristina Malmgren ${ }^{r}$, Savvas Papacostas ${ }^{s}$, Anna Kelement, Markus Reuberu, Eugen Trinkav, Philippe Ryvlinw

a. Department of Neurology, University Hospital of Ioannina, loannina, Greece

b. Adult Department, Danish Epilepsy Center, Epilepsihospitalet, Dianalund, Denmark

c. UCL Queen Square Institute of Neurology, Department of Clinical and Experimental Epilepsy, London, United Kingdom

d. Department of Neurology, Department of Clinical Neurophysiology and MEG, VU University Medical Center, Amsterdam, Netherlands

e. Epilepsy Center, University Medical Center, Freiburg, Germany

f. Moscow Research and Clinical Center for Neuriopsychiatry, Russian National Research Medical University, Moscow, Russia

g. Psychiatry Department, Strasbourg University Hospital, Strasbourg, France

h. National Centre for epilepsy, Oslo University Hospital, Oslo, Norway

i. Division of Clinical Epileptology and Experimental Neurophysiology, "Carlo Besta" Neurological Institute Foundation, Milan, Italy

j. Oberärztin Station Gastaut / IME, Epilepsiezentrum, Kork, Germany

k. Epilepsy Surgery Centre IRCCS NEUROMED, Pozzilli, Italy

I. Refractory Epilepsy Centre at Cliniquesuniversitaires Saint-Luc, University Hospital, Brussels, Belgium

m. EEG/Sleep Laboratory. Department of Neurosciences, Hospital de Santa Maria - CHLN, University Hospital, Lisbon, Portugal

n. Department of Neurology, Hospital Ruber Internacional \& Hospital La Luz, Madrid, Spain

o. Epilepsy Surgery Center, Department of Neurosurgery, St. Ivan Rilski University Hospital, Sofia, Bulgaria

p. Epilepsy center, Department of Neurology, University Hospital, Zagreb, Croatia

q. Department of Neurology, Istanbul University Cerrahpasa Faculty of Medicine, Istanbul, Turkey.

r. Department of Clinical Neuroscience, Institute of Neuroscience and Physiology, Gothenburg University, Göteborg, Sweden 
s. Department of Neurology Clinic B, The Cyprus Institute of Neurology \& Genetics, Cyprus School of Molecular Medicine, Nicosia, Cyprus

t. National Institute of Psychiatry and Neurology, Department of Neurology, University of Pécs, Budapest, Hungary

u. Academic Neurology Unit, Royal Hallamshire Hospital, University of Sheffield, Sheffield, United Kingdom

v. Paracelsus Medical University Salzburg, Department of Neurology, Christian Doppler University Hospital, Salzburg, Austria

w. Department of Clinical Neurosciences, University Hospital of Lausanne (CHUV), Lausanne, Switzerland

1: same contribution

Corresponding author: Dr. Andreas Liampas, University Hospital of loannina, Greece

Mailing address: Stavros Niarchos Str., Ioannina, Greece, 45500

Tel.: +30 2462084636 Fax: -

Mobile phone: +306970400731

E-mail: liampasand@gmail.com 


\begin{abstract}
Background: In cases undergoing epilepsy surgery, postoperative psychogenic nonepileptic seizures (PNES) may be underdiagnosed complicating the assessment of postsurgical seizures' outcome and the clinical management. We conducted a survey to investigate the current practices in the European epilepsy monitoring units (EMUs) and the data that EMUs could provide to retrospectively detect cases with postoperative PNES and to assess the feasibility of a subsequent postoperative PNES research project for cases with postoperative PNES.
\end{abstract}

Methods: We developed and distributed a questionnaire survey to 57 EMUs. Questions addressed the number of patients undergoing epilepsy surgery, the performance of systematic preoperative and postoperative psychiatric evaluation, the recording of sexual or other abuse, the follow-up period of patients undergoing epilepsy surgery, the performance of video-electroencephalogram (EEG) and postoperative psychiatric assessment in suspected postoperative cases with PNES, the existence of electronic databases to allow extraction of cases with postoperative PNES, the data that these bases could provide, and EMUs' interest to participate in a retrospective postoperative PNES project.

Results: Twenty EMUs completed the questionnaire sheet. The number of patients operated every year/per center is $26.7( \pm 19.1)$, and systematic preoperative and postoperative psychiatric evaluation is performed in $75 \%$ and $50 \%$ of theEMUs accordingly. Sexual or other abuse is systematically recorded in one-third of the centers, and the mean follow-up period after epilepsy surgery is $10.5 \pm 7.5$ years. In suspected postoperative PNES, video-EEG is performed in $85 \%$ and psychiatric assessment in $95 \%$ of the centers. An electronic database to allow extraction of patientswith PNES after epilepsy surgery is used in $75 \%$ of the EMUs, and all EMUs that sent the sheet completed expressed their interest to participate in a retrospective postoperative PNES project.

Conclusion: Postoperative PNES is an underestimated and notwell-studied entity. This is a European survey to assess the type of data that the EMUs surgical cohorts could provide to retrospectively detect postoperative PNES. In cases with suspected PNES, 
most EMUs perform video-EEG and psychiatric assessment, and most EMUs use an electronic database to allow extraction of patients developing PNES.

\section{Introduction}

Psychogenic nonepileptic seizures (PNES) are paroxysmal events with motor manifestations and/or disturbances of sensation and responsiveness, which resemble epileptic seizures, without being related to epileptic discharges in the electroencephalogram (EEG) and not due to other readily identifiable pathophysiological changes $[1,2]$.

Psychogenic nonepileptic seizures continue to represent a serious diagnostic challenge, and the diagnostic difficulties are enhanced by the fact that a proportion of patients have epilepsy and PNES concurrently or sequentially. If one condition follows the other, epilepsy is mostly the initial disorder [3, 4]. Consequently, epilepsy can be considered as a risk factor for PNES development [3].

The de novo development of PNES has been described after brain surgery for indications other than refractory epileptic seizures, but, nevertheless, PNES are more likely to occur after epilepsy surgery [5-11].

This phenomenon is often underdiagnosed and underestimated, complicating the assessment of postsurgical seizures' outcome and the clinical management. To obtain a clear picture of the entity, it is important to estimate postoperative PNES prevalence, assess potential risk factors as preoperative psychiatric disease, report the diagnostic procedures used for their detection, and identify characteristic PNES manifestations and associations.

The primary aim of our study was to assess the current practice and the data that the epilepsy monitoring units (EMUs) surgical cohorts could provide to retrospectively detect cases with postoperative PNES. Toward this goal, we developed and distributed a questionnaire to gather information from European EMUs regarding the data that they could provide for postoperative PNES detection. 


\section{Material and methods}

This is a questionnaire-based survey conducted in European EMUs. Epilepsy monitoring units' persons in charge were provided with a formulated questionnaire sheet sent by email.

All EMUs persons in charge were provided with a cover letter to describe the background and the aims of the survey, the names, and addresses of the researchers and explain why the respondent was selected. Nonrespondents to the questionnaire received up to 3 electronic requests to complete the questionnaire.

The questionnaire was built by SM, GR, and PR. The questionnaire was based on the parameters of interest of postoperative PNES to be exploredwith amanageable number of questionswithout eliminating entire domains or important constructs of information [12].

The questionnaire was consisted of 9 questions addressing 1) the number of patients undergoing epilepsy surgery per year; 2 ) the performance of systematic preoperative psychiatric evaluation, answered with yes/no; 3) the performance of systematic postoperative psychiatric evaluation, answered with yes/no; 4) the recording of history of sexual or other abuse, answered with systematically/only reported by patients/ no; 5) the follow-up period of patients undergoing epilepsy surgery; 6 ) the performance of video-EEG in suspected postoperative PNES, answered with yes/no; 7) the postoperative psychiatric assessment in cases with suspected postoperative PNES, answered with yes/ no; 8) the existence of an electronic database of patients undergoing epilepsy surgery allowing extraction of caseswith postoperative PNES and the type of information that these data could supply, as demographics, the age at epilepsy onset, the duration of epilepsy, the epilepsy type, the seizures' frequency, the age at epilepsy surgery, the surgical procedure, the operative side, the surgery complications, the postoperative follow-up period, the postoperative time of development of PNES, the methods used for PNES diagnosis, PNES frequency, manifestations and similarities to preoperative seizures, and the seizures outcome at the time of PNES onset, answered with yes/no (if no, please specify how could these data could be provided); and 9) the interest of EMUs to participate in a postoperative 
PNES project, answered with yes/no (Table 1). Ethical approval was obtained by each participating centre, as required by local regulations.

\section{Results}

Fifty-seven EMUs were contacted, and twenty centers responded and completed the sheet. Twelve centers responded that they could not supply us with the information requested in the questionnaire or were not interested to participate in the survey, and twenty-five centers did not respond.

According to the data sheets completed and returned, approximately $26.7( \pm 19.1)$ patients are operated every year/per center for refractory focal epilepsy.

A systematic preoperative psychiatric evaluation is performed in 15 centers (75\%).

A systematic postoperative psychiatric assessment is performed in 10 centers (50\%). History of any abuse is systematically recorded in 7 centers (35\%), and patients are asked directly for any abuse as part of the regular interview. In 7 centers (35\%), any incidence of abuse is recorded if reported by the patient, but it is not systematically explored during the interview.

The mean follow-up period after epilepsy surgery is $10.5 \pm 7.5$ years.

A video-EEG is performed in cases with suspected postoperative PNES in 17 centers (85\%), and a psychiatric assessment is conducted in cases with suspected PNES in 19 centers (95\%).

An electronic database that would allow to extract all the information required for a subsequent research postoperative PNES project is used in 15 centers (75\%).

All EMUs that completed and returned the questionnaire sheet expressed their interest to participate in a subsequent retrospective postoperative PNES project (Table 2). 


\section{Discussion}

This is the first survey to assess the type and the bulk of data that the EMUs surgical cohorts could provide to retrospectively detect postoperative PNES and assess the feasibility of a subsequent research postoperative PNES project.

Postoperative PNES occur in $1.8 \%$ to $8.8 \%$ of patients undergoing epilepsy surgery with the highest frequency being reported by Glosser (8.8\%) [5-11].

The female gender has been proposed as the main risk factor, followed by the presence of a preoperative psychiatric disorder, mainly depression [10, 11]. Not all EMUs carry out systematic preoperative and postoperative evaluation. Psychiatric evaluation is an essential key for the definition of etiology and diagnosis of PNES and the identification of psychiatric comorbidities [13], and it is rather doubtful whether centers without systematic psychiatric evaluation could participate in a postoperative PNES project.

Traumatic life events, particularly sexual abuse, have been identified as risk factors for PNES [14]. Given the focus on PNES as a comorbidity of epilepsy, trauma and sexual abuse are regarded as less significant etiological risks [15, 16], potentially explaining why only few centers systematically report sexual or any other abuse.

It has been described that most cases with postoperative PNES occur within 6 months after epilepsy surgery $[11,17]$. In a case series study, patients developed psychogenic nonepileptic attacks (PNEA) after the first year following epilepsy surgery even 10 years after the surgery [10]. The fact that there are centers with a shorter than 10 years period of follow-up introduces the risk of cases with PNES being missed in the process of a PNES project.

The definitive diagnosis of PNES is quite difficult and should include all available data. Although there are certain clinical features during events, which could help to distinguish PNES from epileptic seizures, it is not always easy to make a diagnosis of PNES on a clinical ground alone, and video-EEG telemetry remains the gold standard in PNES diagnostic work-up [18-20]. Video-EEG in cases with suspected PNES was conducted in most centers, while of high interest is the finding that psychiatric 
assessment was conducted in cases with suspected PNES in almost all centers (95\%) in accordance with Parra et al. recommendation [7].

The low response rate is the main limitation of our survey. The low response rate does not allow our results to be used to draw conclusions for the whole Europe, but they are still indicative for the current practice in European centers.

Conclusively, the high clinical impact of postoperative PNES recognition and accurate diagnosis is essential for adopting appropriate preventative and therapeutic strategies. Postoperative PNES is an underestimated and not well-studied entity. This is a European survey to assess the type of data that the EMUs surgical cohorts could provide to retrospectively detect postoperative PNES.

\section{Declaration of competing interest}

None of the authors has any conflict of interest to disclose.

\section{Acknowledgements}

Authors aknowledge no funding sources.

\section{References}

[1] Reuber $M$ \& Elger CE. Psychogenic nonepileptic seizures: review and update. Epilepsy \& Behavior. 2003;4(3):205-216. https://doi.org/10.1016/S15255050(03)00104-5

[2] D’Alessio L, Giagante B, Oddo S, Silva WW, Solís P, Consalvo D et al. Psychiatric disorders in patients with psychogenic non-epileptic seizures, with and without comorbid epilepsy. Seizure- European Journal of Epilepsy. 2006;15(5):333-339. https://doi.org/10.1016/j.seizure.2006.04.003

[3] Reuber M, Qurishi A, Bauer J, Helmstaedter C, Fernández G, Widman G et al. Are there physical risk factors for psychogenic non-epileptic seizures in patients with epilepsy? Seizure - European Journal of Epilepsy. 2003;12(8):561-567. https://doi.org/10.1016/S1059-1311(03)00064-5

[4] Devinsky O, Sanchez-Villasenor F, Vazquez B, Kothari M, Alper K, Luciano D. Clinical profile of patients with epileptic and nonepileptic seizures. Neurology 1996;46:15301533. https://doi.org/10.1212/wnl.46.6.1530 
[5] Krahn LE, Rummans TA, Sharbrough FW, Jowsey SG, Cascino GD. Pseudoseizures after epilepsy surgery. Psychosomatics. 1995;36(5):487-493. https://doi.org/10.1016/S0033-3182(95)71630-9

[6] Ney GC, Barr WB, Napolitano C, Decker R, Schaul N. New-onset psychogenic seizures after surgery for epilepsy. Arch Neurol. 1998;55(5):726-730. https://doi.org/10.1001/archneur.55.5.726

[7] Parra J, Iriarte J, Kanner AM, Bergen DC. De novo psychogenic nonepileptic seizures after epilepsy surgery. Epilepsia. 1998;39(5):474-477. https://doi.org/10.1111/j.1528-1157.1998.tb01408.x

[8] Glosser G, Roberts D, Glosser DS. Nonepileptic seizures after resective epilepsy surgery. Epilepsia. 1999;40(12):1750-1754. https://doi.org/10.1111/j.15281157.1999.tb01593.x

[9] Reuber M, Kral T, Kurthen M, Elger CE. New-onset psychogenic seizures after intracranial neurosurgery. Acta Neurochir (Wien). 2002;144(9):901-907. https://doi.org/10.1007/s00701-002-0993-7

[10] Markoula S, de Tisi J, Foong J, Duncan JS. De novo psychogenic nonepileptic attacks after adult epilepsy surgery: an underestimated entity. Epilepsia. 2013. 54(12):e159-162. https://doi.org/10.1111/epi.12404

[11] Asadi-Pooya AA, Asadollahi M, Tinker J, Nei M, Sperling MR. Post-epilepsy surgery psychogenic nonepileptic seizures. Epilepsia. 2016;57(10):1691-1696. https://doi.org/10.1111/epi.13513

[12] Kelley K, Clark B, Brown V, Sitzia J. Good Practice in the Conduct and Reporting of Survey Research. Int J Qual Health Care. 2003;15(3):261-6. https://doi.org/10.1093/intahc/mzg031

[13] Robert CD, LaFrance WC Jr. Psychogenic non-epileptic seizures. Epileptic Disorders. 2016.;18(4):337-343. https://doi.org/10.1684/epd.2016.0873

[14] Reuber M, Howlett S, Khan A, Grunewald RA. Non-epileptic seizures and other functional neurological symptoms: predisposing, precipitating, and perpetuating factors. Psychosomatics. 2007;48(3):230-238. https://doi.org/10.1176/appi.psy.48.3.230

[15] Vincentiis S, Valente KD, Thomé-Souza S, Kuczinsky E, Fiore LA, Negrão N. Risk factors for psychogenic nonepileptic seizures in children and adolescents with epilepsy. Epilepsy \& Behavior. 2006;8(1):294-298. https://doi.org/10.1016/j.yebeh.2005.08.014

[16] Duncan R \& Oto M. Predictors of antecedent factors in psychogenic nonepileptic attacks - Multivariate analysis. Neurology. 2008;71:1000-1005. https://doi.org/10.1212/01.wnl.0000326593.50863.21 
[17] González Otárula KA, Tan YL, Dubeau F, Correa JA, Chang E, Hall JA, Knowlton RC, Kobayashi E. Psychogenic Nonepileptic Seizures in Patients With Surgically Treated Temporal Lobe Epilepsy: Presurgical and De Novo Postsurgical Occurrence. Epilepsy Behav. 2017;75:252-255. https://doi.org/10.1016/j.yebeh.2017.08.015

[18] LaFrance Jr WC \& Plioplys S. Neuropsychiatric disorders: does semiology of psychogenic nonepileptic seizures matter? Nat Rev Neurol. 2012;8(6):302-303. https://doi.org/ 10.1038/nrneurol.2012.79

[19] LaFrance WC Jr, Baker GA, Duncan R, Goldstein LH, Reuber M. Minimum requirements for the diagnosis of psychogenic nonepileptic seizures: a staged approach: a report from the International League Against Epilepsy Nonepileptic Seizures Task Force. Epilepsia. 2013;54(11):2005-2018.

https://doi.org/10.1111/epi.12356

[20] Vega-Zelaya L, Torres CV, Garnes-Camarena O, Ortega GJ, García-Navarrete E, Navas M, Sola RG, Pastor J. Electrocorticographic Evidence and Surgical Implications of Different Physiopathologic Subtypes of Temporal Epilepsy. Clin Neurophysiol. 2014;125(12):2349-57. https://doi.org/ 10.1016/j.clinph.2014.03.027 


\section{EMU}

UCL Queen Square Institute of Neurology, Department of Clinical and Experimental Epilepsy

Department of Neurology, Department of Clinical Neurophysiology and MEG, VU University Medical Center

Epilepsy Center, University Medical Center Moscow Research and Clinical Center for Neuriopsychiatry, Russian National Research Medical University

Psychiatry Department, Strasbourg University Hospital

National Centre for epilepsy, Oslo University

Hospital

Division of Clinical Epileptology and

Experimental Neurophysiology, "Carlo Besta"

Neurological Institute Foundation

Oberärztin Station Gastaut / IME,

Epilepsiezentrum

Epilepsy Surgery Centre IRCCS NEUROMED

Refractory Epilepsy Centre at

Cliniquesuniversitaires Saint-Luc, University

Hospital

EEG/Sleep Laboratory. Department of

Neurosciences, Hospital de Santa Maria -

CHLN, University Hospital

Department of Neurology, Hospital

RuberInternacional\& Hospital La Luz

Epilepsy Surgery Center, Department of

Neurosurgery, St. Ivan Rilski University

Hospital

Epilepsy center, Department of Neurology,

University Hospital

Department of Neurology, Istanbul University

Cerrahpasa Faculty of Medicine

Department of Clinical Neuroscience, Institute

of Neuroscience and Physiology, Gothenburg

University

Department of Neurology Clinic B, The Cyprus

Institute of Neurology \& Genetics, Cyprus

School of Molecular Medicine

National Institute of Psychiatry and Neurology,

Department of Neurology, University of Pécs

Academic Neurology Unit, Royal Hallamshire

Hospital, University of Sheffield, Sheffield,

United Kingdom

Paracelsus Medical University Salzburg, Department of Neurology, Christian Doppler University Hospital
Country (City)

United Kingdom (London)

Netherlands (Amsterdam)

Germany (Freiburg)

Russia (Moscow)

France (Strasbourg)

Norway (Oslo)

Italy (Milan)

Germany (Kork)

Italy (Pozzilli)

Belgium (Brussels)

Portugal (Lisbon)

Spain (Madrid)

Bulgaria (Sofia)

Croatia (Zagreb)

Turkey (Istanbul)

Sweden (Göteborg)

Cyprus (Nicosia)

Hungary (Budapest)

United Kingdom (Sheffield)

Austria (Salzburg)

Table 2. The questionnaire sheet 
How many patients your center estimates for epilepsy surgery are operated per year?

Do you perform a systematic preoperative psychiatric assessment in your patients?

Yes/No

Do you perform a systematic postoperative psychiatric assessment in your patients?

Yes/No

Do you record the history of sexual or other abuses?

Yes/No/Only when reported by the patient

For how long do you follow-up your patients after epilepsy surgery?

In suspected post-operative PNES cases, do you perform video-EEG?

Yes/No

In suspected PNES cases, do you perform psychiatric assessment?

Yes/No

Do you have an electronic database that would allow extracting patients from your epilepsy surgery program who suffered preoperative or postoperative PNES?

Yes/No (in that case please specify)

Would your center (yourself or one of your colleagues) be willing to participate tin a retrospective postoperative PNES project?

Yes/No 